\title{
A Brief Introduction to the Weak Gravity Conjecture
}

\author{
Eran Palti \\ Department of Physics, Ben-Gurion University of the Negev, Beer-Sheva 84105, Israel
}

\begin{abstract}
The Weak Gravity Conjecture proposes that a theory with a gauge field that is coupled to gravity must have charged matter on which gravity acts as the weakest force. It also proposes that there is an intrinsic cutoff on such an effective theory, which corresponds to a tower of charged states, that is set by the strength of the gauge coupling (in Planck units). This article is a brief introduction to the Weak Gravity Conjecture, covering aspects of the motivations for it as well as various extensions and refinements.
\end{abstract}

Keywords: Swampland, Quantum Gravity, String Theory DOI: 10.31526/LHEP.2020.176

\section{INTRODUCTION}

There are four known forces in the universe: the strong and weak forces, electromagnetism, and gravity. The known fundamental matter particles are all charged under at least two of these forces. For example, the right-handed electron carries electromagnetic charge and gravitational charge. These charges can be deduced from the Coulomb forces that two electrons feel when placed a distance $r$ apart. There is a gravitational attractive force

$$
F_{\text {Grav }}=\frac{1}{4 \pi r^{2}}\left(\frac{m_{e}}{\sqrt{2} M_{p}}\right)^{2}
$$

where $m_{e}$ is the electron mass, and $M_{p}$ is the Planck mass. And there is the electromagnetic repulsive Coulomb force

$$
F_{\mathrm{EM}}=\frac{1}{4 \pi r^{2}}\left(g_{E M} q_{e}\right)^{2} .
$$

Here the electric charge is $g_{E M} q_{e}$ which we have chosen to split into a continuous parameter $g_{E M}$ which sets the strength of the electromagentic force, and a quantized charge $q_{e}$ associated to the electron in particular (that is conventionally taken to be one $\left.q_{e}=1\right)$. The numerical values of the charges are

$$
\frac{m_{e}}{\sqrt{2} M_{p}} \sim 10^{-22}, \quad g_{E M} q_{e} \sim 10^{-1}
$$

The electromagnetic force acts roughly 21 orders of magnitude more strongly on the electron than gravity. We can say that of all the forces which act on the electron, gravity is the weakest one 1

So in our universe there exists at least one particle on which gravity acts as the weakest force. Could it have been any different? The Weak Gravity Conjecture (WGC) states that it could not have been [1]. Roughly speaking, it postulates that in any consistent theory of physics which includes gravity, there must exist at least one particle on which gravity acts as the weakest force. Importantly, this does not mean that it is the weakest force acting on all particles. If we convert this statement into parameters which appear in the Lagrangian, we have the statement that in a theory with a gauge field (let us restrict to $U(1)$

${ }^{1}$ Note that there are forces in nature which act on the electron even more weakly, for example the strong force, but this is because the electron is completely uncharged under them and so they have exactly zero action. gauge fields for simplicity), with gauge coupling $g$, there must exist at least one particle with quantized charge $q$ and mass $m$ which satisfies the bound

$$
m \leq \sqrt{2} g q M_{p} .
$$

The bound (4) is termed the Electric WGC. This is to differentiate it from a related bound termed the Magnetic WGC. The simplest way to introduce this second bound is through the electromagnetic dual of (4) [1]. Under electromagnetic duality the gauge coupling $g$ is exchanged for its inverse $g^{-1}$, while the electrically charged particle is exchanged for a monopole with mass $m_{\text {Mon }}$. So we have

$$
m_{\text {Mon }} \leq \frac{\sqrt{2}}{g} M_{p},
$$

where the electric charge is taken to be one for simplicity. The mass of a monopole is at least the energy stored in its magnetic field. This is infinite unless we place a cutoff $\Lambda$ on the effective theory where the description of the monopole as a particle must break down. This cutoff is related to the mass of the monopole through

$$
m_{\mathrm{Mon}} \sim \frac{\Lambda}{g^{2}} .
$$

Combining (5) and (6) we obtain the Magnetic WGC

$$
\Lambda \lesssim g M_{p}
$$

What is the meaning of the cutoff $\Lambda$ ? What type of new physics must appear there? One way to approach this question is to consider how the Electric WGC (4) must behave under renormalization. First, since the gauge coupling and mass both run with energy scale, we should determine at which energy scale should (4) be expected to hold. To violate the bound, we need to show that there is no charged particle in the theory up to the mass scale $\sim g M_{p}$. Therefore, the bound should be evaluated at that scale. Consider the case where the WGC particle satisfying the bound $(4)$ is a scalar ${ }^{2}$ The mass of a scalar runs naturally to the cutoff scale of the theory $\Lambda$. However, the gauge coupling, being a technically natural parameter, is only logarithmically sensitive to the cutoff. The bound (4) therefore only makes sense from a radiative naturalness perspective if (7) is satisfied. What if we want to keep the bound 4 above

\footnotetext{
${ }^{2}$ The case of a fermion follows the same logic by relating the mass of the fermion to the expectation value of a Higgs field through (technically natural) Yukawa couplings.
} 
the scale $g M_{p}$ ? Then we should require that the gauge coupling begins to run with energy scale as a power-law rather than logarithmically above the scale $\Lambda$. The only way to do this is that a tower of charged particles should appear at that scale. Integrating in such a tower leads to the required power-law running of the gauge coupling. This is one motivation for a refined version of the Magnetic WGC which states that the scale $\Lambda$ in $(7)$ is actually the mass scale of an infinite tower of charged particles of increasing masses and charges (such that at each level (4) is satisfied) [2, 3, 4, 5, 6, 7]. So we have

$$
m_{\infty} \sim g M_{p}
$$

with $m_{\infty}$ denoting the mass scale of an infinite tower of particles, so the particle of quantized charge $n$ would have mass $n m_{\infty} 3^{3}$

\section{QUANTUM GRAVITY AND THE WGC}

The Weak Gravity Conjecture is part of the more general Swampland program which aims at determining constraints on effective theories that are requirements for them to potentially have an ultraviolet completion. See [8] for a comprehensive review. The Swampland constraints are associated to the presence of gravity and so with the existence of an ultraviolet completion which includes quantum gravity. The WGC therefore is postulated to have its origin in quantum gravity physics.

What aspects of quantum gravity could require the Electric (4) and Magnetic (7) constraints to hold? The primary piece of evidence is that they appear to hold in String Theory. Since this is a brief introduction, we will not discuss the details of this, and instead refer to the review [8].

Moving away from string theory evidence, there have been various proposals for general quantum gravity reasons for supporting the WGC. At this point it is worthwhile distinguishing between two versions of the Electric WGC. The first, weak version, is that there is some state in the theory satisfying the bound (4) but this state need not be a particle. So its mass can be much larger than the Planck mass and it is best regarded as a Black Hole. An extremal charged black hole will saturate (4) classically at the two-derivative level. The question of whether it will respect the bound even once higher derivative and quantum corrections are introduced it tied to the sign of higher derivative terms. These signs can be bounded within quantum field theory, so-called positivity bounds, and it may be that such constraints would hold also in the presence of gravity. Research in this direction appears to show that the structure of higher derivative terms is such that indeed black holes satisfy the Electric WGC [1, 9, 10, 11, 12, 13, 14, 15, 16, 17, 18.

\subsection{Black Hole discharge and species}

A stronger, and more interesting, version of the WGC is that the bound (4) must hold for a particle. So a state with mass smaller than $M_{p}$. In this case general arguments usually tie in to black hole discharge. That is, for charged extremal black holes to be able to emit charge (a la Hawking radiation), there must

\footnotetext{
${ }^{3}$ The meaning of an infinite tower does not make sense for finite $g$, since this would go to the infinite ultraviolet. It is meant only in the sense of the tower increasing in the number of states as $g \rightarrow 0$, eventually having an infinite number of states below say $M_{p}$.
}

exist an appropriately charged particle. It is simple to show that the requirement on that charge is exactly the Electric WGC [1]. However, it remains an open problem to show that something goes wrong in a theory where black holes cannot discharge.

One approach is that if black holes cannot discharge, then after shedding their mass through Hawking radiation, they will lead to remnants which are stable due to the charge, and that such remnants may violate some principles of quantum gravity such as entropy bounds. However, black holes satisfy an extremality bound

$$
M_{B H} \geq \sqrt{2} g Q_{B H} M_{p},
$$

where $M_{B H}$ is the black hole mass, and $Q_{B H}$ its quantized charge. The bound means that any remnant mass is bounded from below by its charge, and so at finite gauge coupling only a finite number $N_{\text {rem }}$ of remnants can exist below the Planck scale, $N_{\text {rem }} \sim g^{-1}$. It is reasonable that an infinite number of remnants below a finite mass scale is an inconsistent situation, but this only occurs in the $g \rightarrow 0$ limit. Such a limit is censored through the Magnetic WGC since an infinite tower of states becomes massless. Indeed, far from forbidding stable charged particles below $M_{p}$, the Magnetic WGC requires them. From this perspective, the absence of black hole discharge processes does not appear problematic.

Do a finite number of remnants $N_{\text {rem }}$ violate entropy bounds? One way to approach the question is to ask if having a number of species of particles $N$ violates entropy bounds. This is a question that has been studied many times (see the review [8] for reference). In [8, 19, 20], it was proposed that the natural way to understand how $N$ species are consistent with entropy bounds is through the so-called species scale

$$
\Lambda_{s} \sim \frac{M_{p}}{\sqrt{N}}
$$

This is proposed to be the scale at which gravity becomes strongly coupled in the presence of $N$ species of particles. To see the relation to entropy, consider $N$ species of weakly interacting fields at temperature $T$ in a box of size $R$. The energy of the configuration scales as $E \sim N R^{3} T^{4}$, while the entropy behaves as $S \sim N R^{3} T^{3}$. We can increase the temperature until the configuration collapses into a black hole, this maximum temperature limits the entropy as $S \sim N^{\frac{1}{4}}\left(M_{p} R\right)^{\frac{3}{2}}$. The covariant entropy bound [21, 22] states that this should be less than the bounding area of the box and so implies $N<\left(M_{p} R\right)^{2}$. For the value of $N$ which violates this bound, we find the species scale $\Lambda_{S} \sim R^{-1}$. This is the minimum excitation energy scale in the box, and so the species scale censors increasing $N$ any further than this and thereby ensures the entropy bound. We therefore may conclude that again having a large number $N$ of stable states below $M_{p}$ is not problematic, but rather we should decrease the cutoff scale of the theory as the species scale. The $g \rightarrow 0$ limit which corresponds to $N \rightarrow \infty$ is then a limit where gravity becomes strongly coupled at zero energy.

\subsection{Obstruction of global symmetries}

It is believed that there are no exact global symmetries in quantum gravity. The Weak Gravity Conjecture can be viewed as quantifying how approaching a global symmetry limit from a gauge symmetry is obstructed by quantum gravity. There are two ways to view this connection. The first is by noting that 
the weak-coupling limit of a gauge symmetry $g \rightarrow 0$ is similar to 'converting' it into a global symmetry. This is because the propagating gauge field decouples form the theory in that limit, while the symmetry selection rules remain exact. In this sense, the Magnetic WGC can be viewed as a quantum gravity obstruction of this global symmetry limit.

Another relation to global symmetries arises by noting that a pure $U(1)$ gauge theory with no charged matter at all actually has a 1-form global symmetry [23. Schematically, it is the symmetry of gauge transformations $A_{\mu} \rightarrow A_{\mu}+\sigma_{\mu}$, where $\partial_{[v} \sigma_{\mu]}=0$ but $\sigma_{\mu}$ cannot be written as $\partial_{\mu} \lambda$. This global symmetry is broken by adding charged matter to the theory. Therefore, in a theory with no global symmetries such as quantum gravity, it must be that any gauge symmetry should have matter charged under it. This matter can be very massive, but the WGC is in this sense a bound on how massive the charged matter can be.

\subsection{Underlying emergence/unification}

It is possible to rewrite the Magnetic WGC (7) in an illuminating way

$$
\frac{1}{g^{2}} \sim \sum_{n=1}^{N} n^{2}
$$

with

$$
N^{3} \sim\left(\frac{M_{p}}{m_{\infty}}\right)^{2} .
$$

The idea here is that (11) should be thought of as the 1-loop contribution to the running of the gauge coupling coming from integrating out a tower of charged states of increasing charges (and masses) up to $N$. The ultraviolet cutoff of the tower is at $\Lambda \sim N m_{\infty}$ and 12 is chosen such that this coincides with the species scale 10 .

This rewriting suggests an explanation for the Magnetic WGC as the statement that any $U(1)$ gauge theory should become strongly coupled at the species scale, the same scale where gravity is strongly coupled [24]. So as a type of unification. A more radical interpretation is that it is the statement that all $U(1)$ gauge fields are not fundamental but emergent in the infrared from integrating out the tower of states that are associated with the magnetic WGC [25, 6, 8]. This is motivated by the fact that at least in toy models of emergent gauge fields, such as the $C P^{N}$ model [26, 27, 25], the infrared value of the gauge coupling is such that it is dominated by the 1-loop running due to the massive states from which the gauge field emerges. In either interpretation, the idea is that weak coupling limits can only arise in quantum gravity by integrating out towers of charged states down from the species scale, and this implies the existence of the tower of states associated with the Magnetic WGC.

\section{SOME REFINEMENTS OF THE WGC}

The Weak Gravity Conjecture as stated in (4) and (7) applies to a theory with a single $U(1)$ gauge symmetry. However, ideas such as requiring black hole discharge or that gravity should be the weakest force are more general and can be applied to more involved theories. In this section we discuss some of these applications as well as other refinements of the conjectures.

\subsection{Strong versions}

There are various versions of the Electric WGC which refer to the nature of the WGC particle that respects the bound (4). Perhaps the most important is the so-called strong version which states that the particle is the lightest charged particle [1]. The importance of this is that without insisting on at least some similar restriction on its lightness, it is not clear that the Electric WGC could ever constrain an effective field theory as one could always push the mass of the particle above the theory cutoff. In the case of multiple $U(1) \mathrm{s}$, discussed below, this definition in terms of the lightest particle is more subtle. See for example [2. 28]. A reasonable statement which seems to be respected in all known string theory examples is that it is one of the lightest particles, so it cannot be made parametrically heavier than other charged particles.

\subsection{Multiple U(1)s}

The case when there are multiple $U(1)$ gauge symmetries was studied in [29]. It was shown that requiring black holes charged under multiple $U(1)$ symmetries to discharge themselves leads to a stronger condition on the charged matter spectrum than applying to each individual $U(1)$.

The precise statement is as follows. Consider $M U(1)$ symmetries with index $a$ and gauge couplings $g_{a}$. We define a charge vector for a particle under the $U(1)$ symmetries as

$$
\mathbf{q}=\left(g_{1} q_{1}, g_{2} q_{2}, \ldots g_{M} q_{M}\right)
$$

We also require multiple particles and so consider $\mathbf{q}_{i}$ with $i$ ranging over the set of particles with masses $m_{i}$ that are required from the WGC. The charge-to-mass vectors are defined as

$$
\mathbf{z}_{i}=\frac{\mathbf{q}_{i} M_{p}}{m_{i}} .
$$

The WGC statement is then that the convex hull of the vectors (14) must contain the unit ball. This is the requirement that any black holes in the theory can discharge themselves.

The Magnetic WGC was presented such that the scale $g M_{p}$ is of a tower of charged states. In the case of multiple $U(1) \mathrm{s}$, a stronger version of this statement is that the charged states for a lattice, so taking two such charged states and adding their form leads to another charged state. This was proposed as the Lattice WGC in [2], and later refined to allow for only a sublattice, so for some gaps in the lattice [5, 3].

\subsection{Massless scalar fields}

In the case when there are also some massless scalar fields present in the theory, the requirement on the particle in the Electric WGC was argued in [30] to be modified. The idea is that the WGC can be viewed as the statement that placing two such particles near each other, they will self-repel rather than selfattract. The gauge force is a self-repulsive one while the gravitational force is a self-attractive one. The bound (4) is the statement that they should self-repel. There are some arguments for why there should be at least one self-repulsive particle in the theory, for example in [1], but they are not any stronger than the arguments for black hole discharge, and it remains an open problem to prove that a self-repulsive particle must exist. Still, requiring such a particle, it is simple to see that the condition (4) should be modified in the presence of scalar fields which generate an additional self-attractive Coulomb force. 
Let us denote the coupling of the particles to scalar fields by $\mu_{i}$, with $i$ being an index ranging over the massless scalar fields $\phi^{i}$ in the theory. We define the scalar field space metric $g_{i j}$ through the kinetic terms of the scalar fields $g_{i j} \partial \phi^{i} \partial \phi^{j}$, then the modified Electric WGC reads [30]

$$
\left(\sqrt{2} g q M_{p}\right)^{2} \geq m^{2}+g^{i j} \mu_{i} \mu_{j} M_{p}^{2}
$$

where the last term accounts for the additional scalar attractive force. It is useful to note that the coupling $\mu_{i}$ can be written in terms of the dependence of the mass of the WGC particle on the scalar fields as $\mu_{i}=\partial_{\phi^{i}} m$. The proposal (15) was generalised to multiple $U(1) \mathrm{s}$ in [31].

\subsection{Generalisation to $p$-forms}

So far the Weak Gravity Conjecture was applied to gauge fields and charged particles. But it has been proposed to hold more generally such that it applies to general $p$-forms and $p-1$ branes [1, 2]. Defining the coupling strength of the $p$-form as $g_{p}$ through its kinetic term $\frac{1}{2 g_{p}^{2}}\left|F^{(p+1)}\right|^{2}$, the statement is that in a $d$-dimensional theory with a $p$-form field, there must exist a $p-1$ brane with quantized charge $q_{p}$ and tension $T_{p}$ satisfying the bound

$$
\frac{p(d-p-2)}{d-2} T_{p}^{2} \leq g_{p}^{2} q_{p}^{2}\left(M_{p}^{d}\right)^{d-2}
$$

where $M_{p}^{d}$ denotes the $d$-dimensional Planck mass.

The case of 0 -forms, so axions, is special and the statement is that given an axion with decay constant $f$, there must exist an instanton coupling to the axion with action $S$, such that

$$
f S \leq M_{p}
$$

Interestingly, this bound has important implications for models of inflation based on axions which require $f>M_{p}$. Specifically, that in such cases $S<1$ implies that the instanton expansion in large instanton action breaks down, and the theory is not under control. In [32] a cutoff related to axions, so a magnetic WGC type constraint, was proposed.

\subsection{Broken supersymmetry}

In [33], a slight strengthening of the Electric WGC was proposed in relation to supersymmetry. It was argued that the inequality (4) can only be saturated, so be an exact equality, if there is unbroken supersymmetry in the theory. This is a reasonable expectation since supersymmetry is the only local symmetry which can relate the mass of a particle to its charge, and quantum gravity is proposed to have no exact global symmetries.

There is an interesting consequence of this stronger version, when combined with the application to $p$-forms and $p-1$ branes. In string theory, there are many solutions where there is an Anti-de Sitter (AdS) space which is supported by a nonvanishing $p$-form flux. Such backgrounds are known to possess a nonperturbative instability where a $p-1$ brane nucleates and expands out to the boundary of AdS thereby reducing the $p$ form flux by one unit [34]. The presence of such a brane, and the related instability, is guaranteed by the WGC if the background is not supersymmetric. This led to a stronger proposal that any non-supersymmetric AdS background in quantum gravity must exhibit an instability [33. 35]. If true, this would have important consequences within the AdS/CFT correspondence because the instability would manifest in the boundary theory as an instantaneous decay [36], so the boundary theory cannot have a stable vacuum.

\section{SUMMARY}

The Weak Gravity Conjecture is an intriguing proposal that, in quantum gravity, the presence of a gauge symmetry requires certain matter charged under it. The Electric WGC (4) proposes that at least some of this matter should be such that gravity is the weakest force acting on it, while the Magnetic WGC (in its more modern form) (7) proposes that there must actually be an infinite tower of charged matter at a mass scale set by the coupling strength of the gauge symmetry.

The strongest current evidence for the conjectures is that they are respected in all known examples in string theory. There are also some more general quantum gravity arguments, mostly based on black holes, which are indicative but as yet remain incomplete.

We reviewed various proposals for extensions and refinements of the Weak Gravity Conjecture. These led to various interesting consequences, from ruling out certain models of inflation to proposing that non-supersymmetric Conformal Field Theories with AdS duals must be unstable.

\section{ACKNOWLEDGMENTS}

This research was supported by the ISRAEL SCIENCE FOUNDATION (grant No. 741/20).

\section{References}

[1] N. Arkani-Hamed, L. Motl, A. Nicolis, and C. Vafa, JHEP 06, 060 (2007) arXiv:hep-th/0601001.

[2] B. Heidenreich, M. Reece, and T. Rudelius, JHEP 02, 140 (2016). arXiv:1509.06374 [hep-th].

[3] B. Heidenreich, M. Reece, and T. Rudelius, JHEP 08, 025 (2017) arXiv:1606.08437 [hep-th].

[4] D. Klaewer and E. Palti, JHEP 01, 088 (2017). arXiv:1610.00010 [hep-th].

[5] M. Montero, G. Shiu, and P. Soler, JHEP 10, 159 (2016). arXiv:1606.08438 [hep-th].

[6] T. W. Grimm, E. Palti, and I. Valenzuela, JHEP 08, 143 (2018), arXiv:1802.08264 [hep-th].

[7] S. Andriolo, D. Junghans, T. Noumi, and G. Shiu, Fortsch. Phys. 66, 1800020 (2018), arXiv:1802.04287 [hep-th].

[8] E. Palti, Fortsch. Phys. 67, 1900037 (2019). arXiv:1903.06239 [hep-th].

[9] Y. Kats, L. Motl, and M. Padi, JHEP 12, 068 (2007). arXiv:hep-th/0606100.

[10] A. Adams, N. Arkani-Hamed, S. Dubovsky, A. Nicolis, and R. Rattazzi, JHEP 10, 014 (2006), arXiv:hepth/0602178.

[11] C. Cheung and G. N. Remmen, JHEP 12, 087 (2014). arXiv:1407.7865 [hep-th].

[12] C. Cheung and G. N. Remmen, Phys. Rev. Lett. 118, 051601 (2017). arXiv:1608.02942 [hep-th]. 
[13] Y. Hamada, T. Noumi, and G. Shiu, Phys. Rev. Lett. 123, 051601 (2019) arXiv:1810.03637 [hep-th].

[14] B. Bellazzini, M. Lewandowski, and J. Serra, Phys. Rev. Lett. 123, 251103 (2019) arXiv:1902.03250 [hep-th].

[15] W.-M. Chen, Y.-T. Huang, T. Noumi, and C. Wen, Phys. Rev. D 100, 025016 (2019). arXiv:1901.11480 [hep-th].

[16] C. Cheung, J. Liu, and G. N. Remmen, JHEP 10, 004 (2018). arXiv:1801.08546 [hep-th].

[17] C. Cheung, J. Liu, and G. N. Remmen, Phys. Rev. D 100, 046003 (2019) arXiv:1903.09156 [hep-th].

[18] S. Andriolo, T.-C. Huang, T. Noumi, H. Ooguri, and G. Shiu, Phys. Rev. D 102, 046008 (2020) arXiv:2004.13721 [hep-th].

[19] G. Dvali and C. Gomez, Phys. Lett. B 674, 303 (2009). arXiv:0812.1940 [hep-th].

[20] R. Brustein and M. Schmidt-Sommerfeld, JHEP 07, 047 (2013), arXiv:1209.5222 [hep-th].

[21] W. Fischler and L. Susskind, (1998), arXiv:hep-th/9806039

[22] R. Bousso, JHEP 07, 004 (1999) arXiv:hep-th/9905177.

[23] D. Gaiotto, A. Kapustin, N. Seiberg, and B. Willett, JHEP 02, 172 (2015) arXiv:1412.5148 [hep-th].

[24] B. Heidenreich, M. Reece, and T. Rudelius, Eur. Phys. J. C 78, 337 (2018) arXiv:1712.01868 [hep-th].

[25] D. Harlow, JHEP 01, 122 (2016) arXiv:1510.07911 [hep-th]

[26] A. D'Adda, M. Luscher, and P. Di Vecchia, Nucl. Phys. B 146, 63 (1978)

[27] E. Witten, Nuclear Physics B 149, 285 (1979)

[28] P. Saraswat, Phys. Rev. D 95, 025013 (2017). arXiv:1608.06951 [hep-th].

[29] C. Cheung and G. N. Remmen, Phys. Rev. Lett. 113, 051601 (2014). arXiv:1402.2287 [hep-ph].

[30] E. Palti, JHEP 08, 034 (2017) arXiv:1705.04328 [hep-th].

[31] B. Heidenreich, M. Reece, and T. Rudelius, JHEP 10, 055 (2019) arXiv:1906.02206 [hep-th].

[32] A. Hebecker and P. Soler, JHEP 09, 036 (2017). arXiv:1702.06130 [hep-th].

[33] H. Ooguri and C. Vafa, Adv. Theor. Math. Phys. 21, 1787 (2017). arXiv:1610.01533 [hep-th].

[34] J. M. Maldacena, J. Michelson, and A. Strominger, JHEP 02, 011 (1999) arXiv:hep-th/9812073.

[35] B. Freivogel and M. Kleban, (2016), arXiv:1610.04564 [hep-th].

[36] G. T. Horowitz, J. Orgera, and J. Polchinski, Phys. Rev. D 77, 024004 (2008) arXiv:0709.4262 [hep-th] 